Please do not remove this page

RMIT

UNIVERSITY

\title{
Polychromatic gap solitons and breathers in nonlinear waveguide arrays
}

Sukhorukov, Andrey; Neshev, Dragomir; Dreishuh, Alexander; Fischer, Robert; Ha, Sangwoo; Krolikowski, Wieslaw; Bolger, Jeremy

https://researchrepository.rmit.edu.au/esploro/outputs/9921863447501341/filesAndLinks?institution=61RMIT_INST\&index=null

Sukhorukov, A., Neshev, D., Dreishuh, A., Fischer, R., Ha, S., Krolikowski, W., Bolger, J., Eggleton, B., Mitchell, A., Austin, M., \& Kivshar, Y. (2007). Polychromatic gap solitons and breathers in nonlinear waveguide arrays. 2007 Bragg Gratings, Photosensitivity and Poling in Glass Waveguides (BGPP) and Nonlinear Photonics (NP) Topical Meeting, 13058-13076.

https://researchrepository.rmit.edu.au/discovery/fulldisplay/alma9921863447501341/61RMIT_INST:Resea rchRepository

Document Version: Published Version

Repository homepage: https://researchrepository.rmit.edu.au

(c) 2007 Optical Society of America

Downloaded On 2023/04/26 12:58:53 +1000

Please do not remove this page 


\title{
Polychromatic gap solitons and breathers in nonlinear waveguide arrays
}

\author{
Andrey A. Sukhorukov ${ }^{1}$, Dragomir N. Neshev ${ }^{1}$, Alexander Dreischuh ${ }^{1,2}$, \\ Robert Fischer ${ }^{1}$, Sangwoo $\mathbf{H a}^{1}$, Wieslaw Krolikowski ${ }^{1}$, Jeremy Bolger ${ }^{3}$, \\ Benjamin J. Eggleton ${ }^{3}$, Arnan Mitchell ${ }^{4}$, Michael W. Austin ${ }^{4}$, and Yuri S. Kivshar ${ }^{1}$ \\ Centre for Ultra-high bandwidth Devices for Optical Systems (CUDOS), \\ ${ }^{1}$ Nonlinear Physics Centre and Laser Physics Centre, \\ RSPhysSE, Australian National University, Canberra, Australia; \\ Email: ans124@rsphysse.anu.edu.au; Tel: +61-2-6125-3423; Fax: +61-2-6125-8588 \\ ${ }^{2}$ Department of Quantum Electronics, Faculty of Physics, Sofia University, 1164 Sofia, Bulgaria \\ ${ }^{3}$ School of Physics, University of Sydney, Sydney NSW 2006, Australia \\ ${ }^{4}$ School of Electrical and Computer Engineering, \\ RMIT University, Melbourne VIC 3001, Australia
}

We predict the spatial localization of multiple wavelength components in the form of stationary polychromatic gap solitons and dynamic multi-gap breathers, and observe experimentally tunable spatio-spectral trapping of supercontinuum radiation in nonlinear periodic photonic structures.

(C) 2007 Optical Society of America

OCIS codes: $190.4420,190.5940$

Photonic structures with a periodic modulation of the optical refractive index open novel possibilities for designing the fundamental aspects of optical wave dynamics. The physics of light propagation in periodic photonic structures is governed by the scattering of waves from the high refractive index regions and the subsequent interference of the scattered waves. This is a resonant process, which is sensitive to both frequency and propagation angle. As such, periodic photonic structures find various applications, including spatial beam control and manipulation of beam refraction and diffraction. These applications, however, are primarily optimized for beam shaping and deflection in a narrow-frequency range. In many practical cases, including ultra-broad bandwidth optical communications or propagation of ultra-short (sub-10 fs) pulses, the bandwidth of optical signals can span over a wide frequency range.

This work suggests theoretically and demonstrates experimentally novel possibilities for all-optical spatial switching and simultaneous spectral reshaping of light beams of ultra-broad spectral bandwidth by the combined effects of material periodicity and incoherent interaction of multiple colors in a nonlinear waveguide array. For the first time to our knowledge, we observe the simultaneous spatio-spectral localization of supercontinuum radiation and the formation of supercontinuum gap solitons. Our results demonstrate a novel scheme for tunable reshaping of polychromatic beams by periodic photonic structures.

(a)

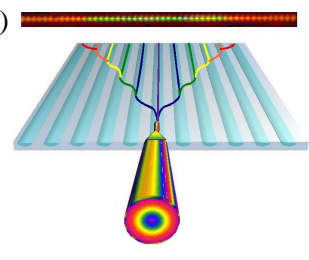

(b)

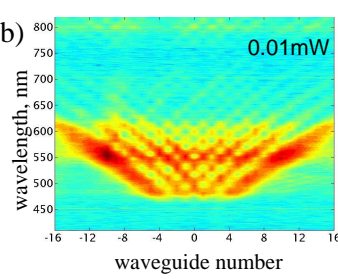

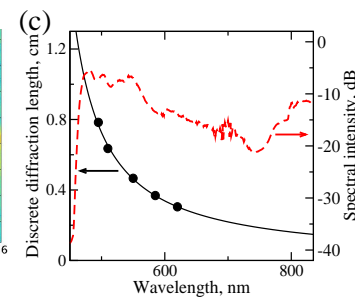
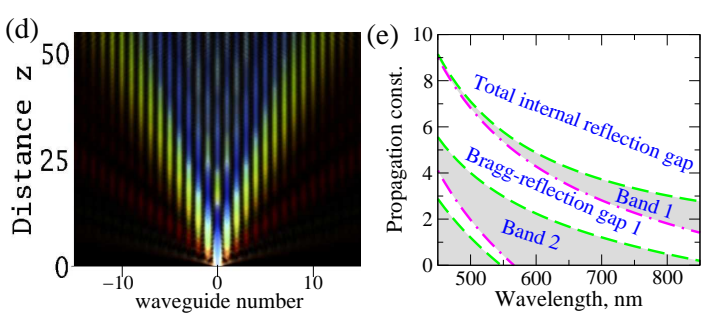

Fig. 1. (a) Schematic of coupling of the supercontinuum to an array of optical waveguides, where different spectral components are redistributed in different waveguides. (b) Measured spectrally resolved output (log scale) from polychromatic discrete diffraction at low average power. (c) Supercontinuum spectrum (red) and measured dispersion of the waveguide coupling length (black). (d) Numerical simulation of linear polychromatic propagation inside the array and spatial separation of colours. (e) Calculated band-gap diagram.

We consider the propagation of polychromatic beams through an array of nonlinear optical waveguides [Fig. 1(a)]. In experiments, multi-color beams combining the properties of high spectral brightness and spatial coherence can be readily generated in photonic-crystal fibers ${ }^{1}$. When the beam is coupled to a single waveguide at the input, at low optical powers (linear regime) each spectral component exhibits discrete diffraction where the light is concentrated into the wings of the beam rather than in its center. Typical polychromatic diffraction pattern is shown in Fig. 1(a), and the corresponding spectrally resolved intensity distribution in each output channel (measured by a spectrometer for the waveguide arrays sample fabricated by Titanium indiffusion in a X-cut $55 \mathrm{~mm}$ long mono-crystal Lithium 


\section{JMB5.pdf}

Niobate wafer) is shown in Fig. 1(b). The beam spreading is characterized by the discrete diffraction length, which defines the distance where the diffraction pattern is extended by two extra waveguides. The diffraction length varies in our experimental sample from $1 \mathrm{~cm}$, for blue, to less than $0.4 \mathrm{~cm}$, for red spectral regions [Fig. 1(c, black curve)]. As a result, the red components dominate in the beam wings, while the blue components are dominant in the central region. This effect of color separation is somewhat analogous to the superprism phenomena observed in photonic crystals $^{2}$. This observation agrees with the numerical simulation of supercontinuum light diffraction in the waveguide array shown in Fig. 1(d).
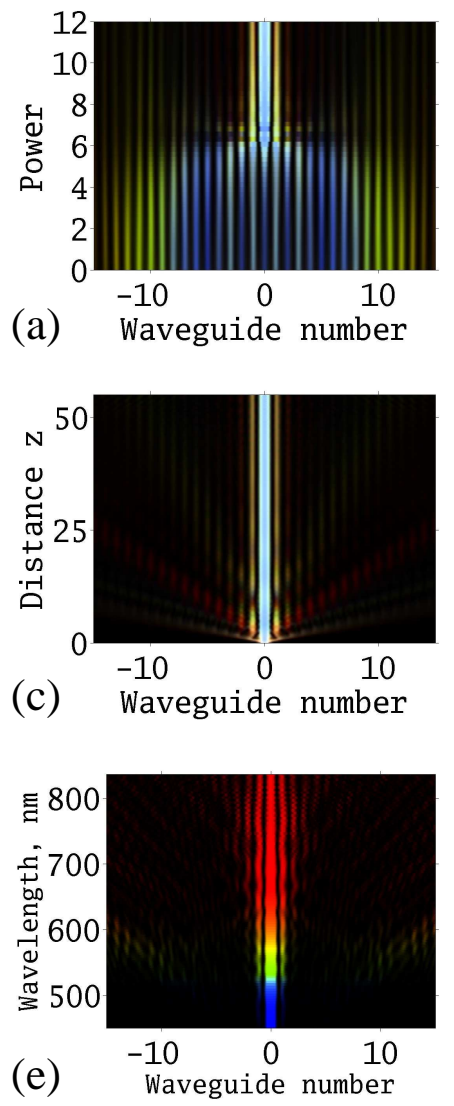

Fig. 2. (a-b) Numerically calculated dependence of the output beam characteristics on the input supercontinuum power: (a) transformation of the output beam profile, and (b) the fraction of output power remaining at the central waveguide for different spectral components. (c-f) Soliton formation for the power level $P_{0} \simeq 12$. Shown are (c) the beam propagation dynamics inside the array, (d) Propagation constants vs. the wavelength, dashed and dashed-dotted lines mark the band edges as indicated in Fig. 1(e), (e) spectrally resolved output intensity profile, and (f) output phase profiles of individual spectral components.
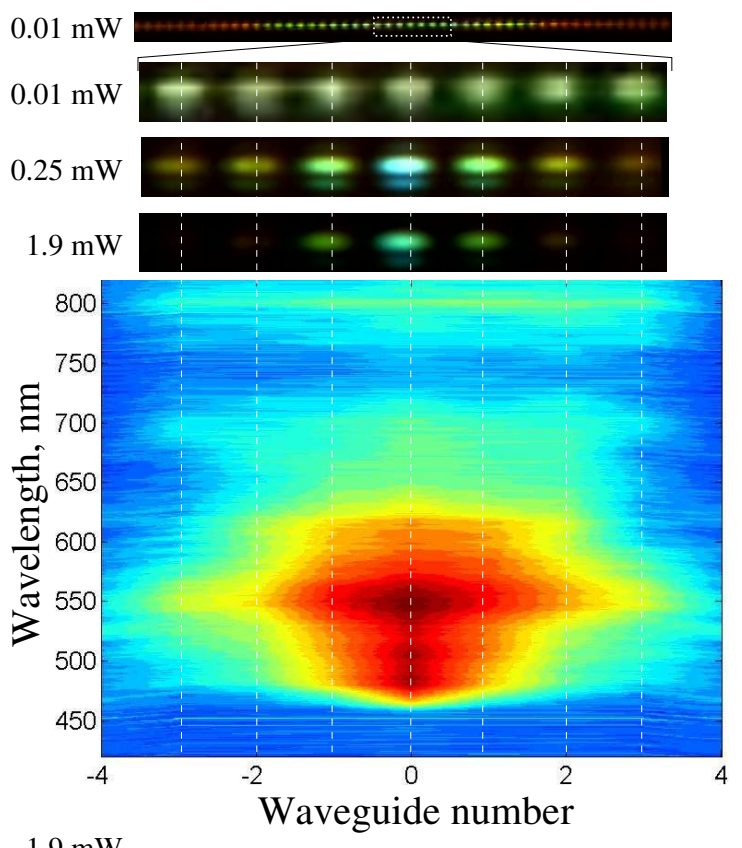

$1.9 \mathrm{~mW}$

Interf.

Fig. 3. Experimental observation of spatiospectral supercontinuum beam reshaping in the nonlinear waveguide array: (a-c) Real-color CCD camera images of the output beam intensity profile: (a) Diffraction profile at low power; (b,c) Nonlinear localization with increasing supercontinuum power and formation of polychromatic gap soliton (c). (d) Interferogram of the output beam profile (c) and a reference supercontinuum beam, integrated over all spectral components. (e,f) Spectrally resolved measurements of the profiles (a) and (c), respectively.

We now investigate the possibility to suppress the diffraction-induce broadening and separation of spectral components through the nonlinear beam self-action. Specifically, at high laser powers, the spectral components can interact incoherently with each other (no new frequency components are generated) due to the intensity-dependent change of the optical refractive inde ${ }^{3}$ through the photovoltaic effect. An important characteristic of the photovoltaic nonlinearity in Lithium Niobate is that an increase of the beam intensity leads to a local decrease of the material refractive index ${ }^{4}$. We model the nonlinear propagation and interaction of all spectral components by including a nonlinear spectral response into the refractive index modulation $\Delta n$.

Although the negative nonlinear response would result in self-defocusing and accelerated beam broadening in bulk media, our numerical simulations show that the input beam can experience self-trapping in the form of a supercontinuum gap soliton, see Fig. 2. Note a sharp transition between the regimes of diffraction and soliton formation, associated with collective localization of spectral components from blue to red and infrared [Figs. 2(a) and 2(b)]. Lower degree of 


\section{JMB5.pdf}

localization of red components is due to stronger diffraction and effectively weaker nonlinearly-induced potential that is inversely proportional to the wavelength. The formation of such self-localized beams may become possible solely due to the Bragg scattering from the periodic structure and the simultaneous localization of all spectral components in the photonic bandgap 5 . We perform Fourier analysis of the supercontinuum soliton [Fig. 2(c)] and confirm that the propagation constants of all wavelength components are located inside the first Bragg-reflection gap [Fig. 2(d)], which enables their simultaneous localization [Fig. 2(e)]. Another signature of band-gap trapping is the appearance of the staggered phase structure of each individual spectral components [Fig. 2(f)]. Hence, such localisation represents a uniquely different physical picture compared to the theoretically studied white-light solitons in lattices supported by a focusing nonlinearity ${ }^{6}$.

We observe experimentally simultaneous dynamic spatio-spectral reshaping of the supercontinuum light as its input power is increased [Figs. 3(a-c)]. At certain input power the beam forms a polychromatic soliton which is localised primarily in a single waveguide of the array [Fig. 3(c)]. The important characteristic of this localization process is the fact that it combines all wavelength components (from blue to red) of the supercontinuum spectrum [Fig. 3(e)] and as such achieves suppression of the spatial dispersion in the nonlinear regime through the formation of a polychromatic gap soliton. We note that the existence of spatial gap solitons excited by monochromatic light relies on a special staggered phase structure ${ }^{7-9}$ required for the efficient Bragg scattering. Taking advantage of the high spatial coherence of the supercontinuum light, we perform white-light interferometric measurement of the localized output profile. The observed interference pattern [Fig. 3(d)] reveals that the dominating spectral components in the adjacent waveguides are out-of-phase, providing confirmation that the localized beam is indeed a polychromatic gap soliton. The development of the well-defined staggered phase structure in different spectral components demonstrates an opportunity for nontrivial phase control of supercontinuum radiation, and distinguishes such nonlinear processes from the localisation of spatially incoherent sources ${ }^{6}$.

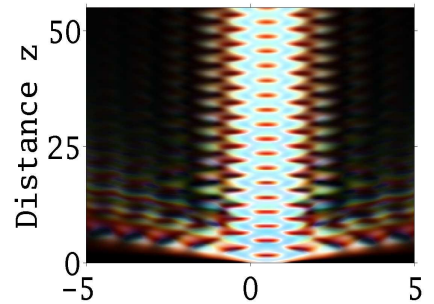

(a)

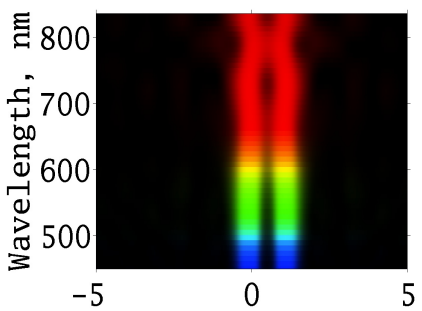

(b)

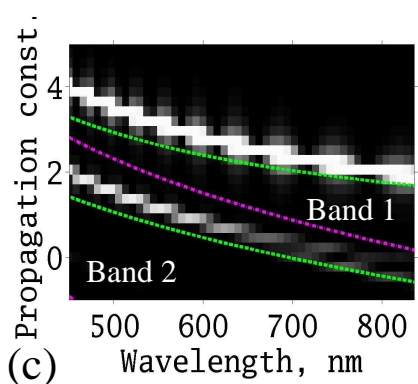

(c) Wavelength, $\mathrm{nm}$

Fig. 4. Numerically calculated polychromatic multi-gap breather in a photonic lattice with self-focusing nonlinearity: (a) The beam propagation dynamics, (b) spectrally resolved output intensity profile, and (c) Propagation constants vs. the wavelength, dashed and dashed-dotted lines mark the band edges as indicated in Fig. 1(e).

Finally, we predict a fundamentally different localization regime, where the individual profiles of all spectral components oscillate periodically along the propagation direction. It was shown that dynamically oscillating multi-gap breathers can be generated by coherent laser light ${ }^{10}$. The oscillation period depends both on the input excitation and the structure of the band-gap spectrum ${ }^{11}$. Since the band-gap spectrum strongly depends on the wavelength, it remained an open question whether multi-gap polychromatic breather can exist. Our simulation results presented in Fig. 4(a) and 4(b) demonstrate, for the first time to our knowledge, that nonlinearity can synchronize the dynamical oscillations of different wavelength components in a broad spectral region. The oscillations occur due to the beating of modes from the total internal reflection and Bragg-reflection gaps, see Fig. 4(c). A distinguishing feature is that the spectral spacing between the two modes is the same for different spectral components from blue to red. This is a nontrivial effect, which occurs through the nonlinear interaction compensating for the strong wavelength dependence of the band-gap structure.

\section{References}

1. P. St. J. Russell, Science 299, 358 (2003).

2. H. Kosaka, T. Kawashima, A. Tomita, M. Notomi, T. Tamamura, T. Sato, and S. Kawakami, J. Lightwave Technol. 17, 2032 (1999).

3. M. Mitchell and M. Segev, Nature 387, 880 (1997).

4. G. C. Valley, M. Segev, B. Crosignani, A. Yariv, M. M. Fejer, and M. C. Bashaw, Phys. Rev. A 50, R4457 (1994).

5. K. Motzek, A. A. Sukhorukov, and Yu. S. Kivshar, Opt. Express 14, 9873 (2006).

6. R. Pezer, H. Buljan, G. Bartal, M. Segev, and J. W. Fleischer, Phys. Rev. E 73, 056608 (2006).

7. J. W. Fleischer, T. Carmon, M. Segev, N. K. Efremidis, and D. N. Christodoulides, Phys. Rev. Lett. 90, 023902 (2003).

8. D. Mandelik, R. Morandotti, J. S. Aitchison, and Y. Silberberg, Phys. Rev. Lett. 92, 093904 (2004).

9. D. Neshev, A. A. Sukhorukov, B. Hanna, W. Krolikowski, and Yu. S. Kivshar, Phys. Rev. Lett. 93, 083905 (2004).

10. D. Mandelik, H. S. Eisenberg, Y. Silberberg, R. Morandotti, and J. S. Aitchison, Phys. Rev. Lett. 90, 253902 (2003).

11. A. Fratalocchi, G. Assanto, K. A. Brzdakiewicz, and M. A. Karpierz, Opt. Express 13, 1808 (2005). 